\title{
Interval - Valued Differential Equations with Generalized Derivative
}

\author{
Natalia Skripnik \\ Department of Optimal Control \& Economic Cybernetics, Odessa National University named after I.I. Mechnikov, Odessa, 65026, \\ Ukraine
}

\begin{abstract}
In this paper the concept of generalized differentiability (proposed in [17]) for interval-valued mappings is used. The interval-valued differential equations with generalized derivative are considered and the existence theorem is proved.
\end{abstract}

Keywords Interval-valued Mapping, Generalized Derivative, Differential Equations, Existence Theorem

\section{Introduction}

Lately the development of calculus in metric spaces became an object of attention of many researchers [1,11-14,16,18 and ref. herein ]. Earlier F.S. de Blasi and F. Iervolino begun studying of set-valued differential equations (SDEs) in semilinear metric spaces[5-8]. Now it transformed into the theory of SDEs as an independent discipline. The properties of solutions, the impulse SDEs, control systems and asymptotic methods for SDEs were considered. On the other hand, SDEs are useful in other areas of mathematics. For example, SDEs are used as an auxiliary tool to prove the existence results for differential inclusions. Also, one can employ SDEs in the investigation of fuzzy differential equations. Moreover, SDEs are a natural generalization of usual ordinary differential equations in fin ite (or infinite) dimensional Banach spaces.

However all these equations have a natural lack - the diameter of a set-valued solution is a non-decreasing function. Possibly it is connected with the fact that these differential equations were entered by analogy with the single-valued theory.

But in the theory of ordinary differential equations a solution in any moment of time is a point (so a solution does not possess the property of "thickness"). Therefore, the similar introduction of the differential equations for a set-valued case is not absolutely adequate.

In[17] a new concept of a derivative of a set-valued mapping that generalizes the concept of Hukuhara derivative was entered and a new type of a set-valued differential equation such that the diameter of its solution can whether

* Corresponding author:

talie@ukr.net (Natalia Skripnik)

Published online at http://journal.sapub.org/am

Copyright (C) 2012 Scientific \& Academic Publishing. All Rights Reserved increase or decrease (for example, to be periodic) was considered. In the ideological sense this definition of the derivative is close to the definitions proposed in[2-4,15].

In this paper the interval-valued differential equations with generalized derivative are considered and the existence theorem is proved.

\section{The Generalized Derivative}

Let $\operatorname{conv}(\mathrm{R})$ be a space of all nonempty closed intervals $X=\left[x_{1}, x_{2}\right] \subset \mathrm{R}$ with Hausdorff metric

$$
h\left(\left[x_{1}, x_{2}\right],\left[y_{1}, y_{2}\right]\right)=\max \left\{\left|y_{1}-x_{1}\right|,\left|y_{2}-x_{2}\right|\right\} \text {. }
$$

Definition 1 [10]. Let $X, Y \in \operatorname{conv}(\mathrm{R})$. A set $Z \in \operatorname{conv}(\mathrm{R})$ such that $X=Y+Z$ is called a $H \mu$ kuhara difference of the sets $X$ and $Y$ and is denoted by $X \frac{n}{-} Y$.

Let $\operatorname{diam} X=x_{2}-x_{1}$. The Hukuhara difference of the sets $X=\left[x_{1}, x_{2}\right]$ and $Y=\left[y_{1}, y_{2}\right]$ exists iff $\operatorname{diam} X \geq \operatorname{diam} Y$ and is equal to $\left[x_{1}-y_{1}, x_{2}-y_{2}\right]$.

Let $X: I \rightarrow \operatorname{conv}(\mathrm{R})$ be an interval-valued mapping; $\left(t_{0}-\Delta, t_{0}+\Delta\right) \subset I$ be a $\Delta$ - neighbourhood of a point $t_{0} \in I ; \Delta>0$.

For any $t \in\left(t_{0}-\Delta, t_{0}+\Delta\right)$ consider the following Hukuhara differences if these differences exist.

$$
\begin{aligned}
& X(t) \stackrel{h}{-} X\left(t_{0}\right), t \geq t_{0}, \\
& X\left(t_{0}\right) \frac{h}{-} X(t), t \geq t_{0}, \\
& X\left(t_{0}\right) \frac{h}{-} X(t), t \leq t_{0}, \\
& X(t) \frac{h}{-} X\left(t_{0}\right), t \leq t_{0}
\end{aligned}
$$

The differences (1) and (2) [(3) and (4)] a re called the right [left] differences. From the definition of the Hukuhara difference it follows that both one-sided differences exist only in the case when $X(t) \equiv F+\{f(t)\}$ for $t \in\left[t_{0}, t_{0}+\Delta\right)$ or 
$t \in\left(t_{0}-\Delta, t_{0}\right]$. If all differences (1) - (4) exist then $X(t) \equiv F+\{f(t)\}$ in $\Delta$-neighbourhood of the point $t_{0}$.

If for all $t \in\left(t_{0}-\Delta, t_{0}+\Delta\right)$ there exists only one of the one-sided differences, then using the properties of the $\mathrm{Hu}$ kuhara difference, we get that the mapping $\operatorname{diam} X: I \rightarrow \mathrm{R}_{+}$in the $\Delta$-neighbourhood of the point $t_{0}$ can be:

a) non-decreasing on $\left(t_{0}-\Delta, t_{0}+\Delta\right)$;

b) non-increasing on $\left(t_{0}-\Delta, t_{0}+\Delta\right)$;

c) non-decreasing on $t \in\left(t_{0}-\Delta, t_{0}\right)$ and non-increasing on $\left(t_{0}, t_{0}+\Delta\right)$;

d) non-increasing on $t \in\left(t_{0}-\Delta, t_{0}\right)$ and non-decreasing on $\left(t_{0}, t_{0}+\Delta\right)$.

Hence, for each of the above mentioned cases only one of combinations of differences is possible:

a) (1) and (3); b) (2) and (4); c) (2) and (3); d) (1) and (4).

Consider four types of limits corresponding to one of the difference types:

$$
\begin{array}{r}
\lim _{t \downarrow_{0}} \frac{1}{t-t_{0}}\left(X(t) \frac{h}{X} X\left(t_{0}\right)\right) ; \\
\lim _{t \downarrow_{0}} \frac{1}{t-t_{0}}\left(X\left(t_{0}\right) \frac{h}{-} X(t)\right) ; \\
\lim _{t \uparrow t_{0}} \frac{1}{t_{0}-t}\left(X\left(t_{0}\right) \frac{h}{-} X(t)\right) ; \\
\lim _{t \uparrow t_{0}} \frac{1}{t_{0}-t}\left(X(t) \frac{h}{-} X\left(t_{0}\right)\right) .
\end{array}
$$

So it is possible to say that in the point $t_{0}$ not more than two limits can exist (as we assumed that there exist only two of four Hukuhara differences).

Considering all above we have that there exist only following comb inations of limits:

a) (5) and (7); b) (6) and (8); c) (6) and (7); d) (5) and (8).

Definition 2. If the corresponding two limits exist and are equal we will say that the mapping $X(\cdot)$ is differentiable in the generalized sense in the point $t_{0}$ and denote the generalized derivative by $D X\left(t_{0}\right)$.

Let us say that the interval-valued mapping $X: I \rightarrow \operatorname{conv}(\mathrm{R})$ is differentiable in the generalized sense on the interval $I$ if it is differentiable in the generalized sense at every point of this interval.

Definition 3. The interval-valued mapping $X: I \rightarrow \operatorname{conv}(\mathrm{R})$ is called absolutely continuous on the interval $I$ if there exist a measurable interval-valued mapping $F(t)$ and a system of intervals $\left[t_{i}, t_{i+1}\right], i=\overline{0, m}, t_{m+1}=T$ such that for all $t \in\left[t_{i}, t_{i+1}\right], i=\overline{0, m}$

$$
X(t)=X\left(t_{i}\right)-\int_{t_{i}}^{h} F(s) d s \text { or } X(t)=X\left(t_{i}\right)+\int_{t_{i}}^{t} F(s) d s .
$$

\section{Differential Equations with the Generalized Derivative}

Consider the differential equation with the generalized derivative

$$
\begin{aligned}
& D X(t)=\Phi(\varphi(t)) F(t, X(t)), \\
& X\left(t_{0}\right)=X_{0},
\end{aligned}
$$

where $t \in\left[t_{0}, T\right] ; X:\left[t_{0}, T\right] \rightarrow \operatorname{conv}(\mathrm{R}), F:\left[t_{0}, T\right] \times \operatorname{conv}(\mathrm{R})$ $\rightarrow \operatorname{conv}(\mathrm{R})$ are interval valued mappings; $X_{0} \in \operatorname{conv}(\mathrm{R})$; $\varphi:\left[t_{0}, T\right] \rightarrow \mathrm{R}$ is a continuous function, function $\Phi(\varphi)=\left\{\begin{array}{l}1, \varphi \neq 0, \\ 0, \varphi=0 .\end{array}\right.$

Definition 4. An interval-valued mapping $X:\left[t_{0}, T\right] \rightarrow \operatorname{conv}(\mathrm{R})$ is called the solution of differential equation (9) if it is absolutely continuous, satis fies (9) almost everywhere on $\left[t_{0}, T\right]$ and

$$
\operatorname{diam} X(t)=\left\{\begin{array}{l}
\text { increases if } \varphi(t)>0, \\
\text { is constant if } \varphi(t)=0, \\
\text { decreases if } \varphi(t)<0 .
\end{array}\right.
$$

If on the interval $\left[\tau_{1}, \tau_{2}\right]$ the function $\varphi(t)>0$, then we have $D X(t)=F(t, X(t))$ and $\operatorname{diam} X(t)$ increases. So $X(t)$ satisfies the integral equation

$$
X(t)=X\left(\tau_{1}\right)+\int_{\tau_{1}}^{t} F(s, X(s)) d s \text { for } t \in\left[\tau_{1}, \tau_{2}\right] .
$$

If on the interval $\left[\tau_{1}, \tau_{2}\right]$ the function $\varphi(t)<0$, then we have $D X(t)=F(t, X(t))$ and $\operatorname{diam} X(t)$ decreases. Therefore $X(t)$ satis fies the integral equation i.e.

$$
\begin{aligned}
& X\left(\tau_{1}\right)=X(t)+\int_{\tau_{1}}^{t} F(s, X(s)) d s, \\
& X(t)=X\left(\tau_{1}\right) \frac{h}{t} \int_{\tau_{1}}^{t} F(s, X(s)) d s .
\end{aligned}
$$

If on the interval $\left[\tau_{1}, \tau_{2}\right]$ the function $\varphi(t) \equiv 0$, then we have $X(t) \equiv X\left(\tau_{1}\right)$.

Example 1. Consider the following differential equation with generalized derivative

$$
\begin{aligned}
& D X(t)=\Phi(\sin t)[1,3], \\
& X(0)=[2,4] .
\end{aligned}
$$

As $\sin t>0$ for $t \in(0, \pi)$ we have

$$
X(t)=[2,4]+\int_{0}^{t}[1,3] d s=[2,4]+[t, 3 t]=[2+t, 4+3 t]
$$

for $t \in\lceil 0, \pi\rceil$.

So for $t=\pi$ we get $X(\pi)=\lceil 2+\pi, 4+3 \pi\rceil$.

Further as $\sin t<0$ for $t \in(\pi, 2 \pi)$ we have 


$$
\begin{aligned}
X(t) & =[2+\pi, 4+3 \pi] \frac{h}{\int_{\pi}^{t}}[1,3] d s= \\
& =[2+\pi, 4+3 \pi] \frac{h}{t} \int_{\pi}^{t} d s[1,3]= \\
& =[2+\pi, 4+3 \pi] \frac{h}{-}(t-\pi)[1,3]= \\
& =[2+\pi, 4+3 \pi] \frac{h}{-}[t-\pi, 3 t-3 \pi]= \\
& =[2+2 \pi-t, 4+6 \pi-3 t] .
\end{aligned}
$$

So for $t=2 \pi$ we get $X(2 \pi)=\lceil 2,4\rceil$.

If we consider this equation for $t \in[0, \infty)$ we will get the periodic solution.

Example 2. Consider the same differential equation with generalized derivative but with $\varphi(t)=\cos t$ :

$$
\begin{aligned}
& D X(t)=\Phi(\cos t)[1,3], \\
& X(0)=[2,4] .
\end{aligned}
$$

As $\cos t>0$ for $t \in\left(0, \frac{\pi}{2}\right)$ then we have

$$
X(t)=[2,4]+\int_{0}^{t}[1,3] d s=[2,4]+[t, 3 t]=[2+t, 4+3 t]
$$

for $t \in\left[0, \frac{\pi}{2}\right]$.

Further as $\cos t<0$ for $t \in\left(\frac{\pi}{2}, \frac{3 \pi}{2}\right)$ then we get

$$
\begin{aligned}
X(t) & =\left[2+\frac{\pi}{2}, 4+\frac{3 \pi}{2}\right] \frac{h}{\int_{\frac{\pi}{2}}^{t}}[1,3] d s= \\
& =\left[2+\frac{\pi}{2}, 4+\frac{3 \pi}{2}\right] \frac{h}{-} \int_{\frac{\pi}{2}}^{t} d s[1,3]= \\
& =\left[2+\frac{\pi}{2}, 4+\frac{3 \pi}{2}\right] \frac{h}{-}\left(t-\frac{\pi}{2}\right)[1,3]= \\
& =\left[2+\frac{\pi}{2}, 4+\frac{3 \pi}{2}\right] \frac{h}{-}\left[t-\frac{\pi}{2}, 3 t-\frac{3 \pi}{2}\right]= \\
& =[2+\pi-t, 4+3 \pi-3 t] .
\end{aligned}
$$

So for $t=1+\pi<\frac{3 \pi}{2}$ we have

$$
\begin{aligned}
X(1+\pi) & =[2+\pi-(1+\pi), 4+3 \pi-3(1+\pi)]= \\
& =[1,1]=\{1\} .
\end{aligned}
$$

It means that the solution exists only for $t \in\lceil 0,1+\pi\rceil$.

Remark. It is obvious that the mapping $F(t, X)$ defines only on "how much" the interval-valued mapping $X(\cdot)$ changes in case of its "decrease" or "increase" and function $\varphi(\cdot)$ defines what will be with $X(\cdot)$ ["decrease" or "increase"]. If $\varphi(t) \equiv 0$ irrespective of $F(t, X)$ the mapping $X(\cdot)$ will be constant.

Example 3. Consider the differential equation from Example 1 with $\varphi(t) \equiv 0$ for $t \in[0,2 \pi]$. Then $X(t) \equiv[2,4]$ for $t \in[0,2 \pi]$.

If we take $\varphi(t) \equiv-1$ then we will have

$$
\begin{aligned}
X(t) & =[2,4] \frac{h}{-} \int_{0}^{t}[1,3] d s= \\
& =[2,4] \frac{h}{-}[1,3] \int_{0}^{t} d s= \\
& =[2,4] \frac{h}{-}[t, 3 t]= \\
& =[2-t, 4-3 t] .
\end{aligned}
$$

Then for $t=1$ we get $X(1)=[1,1]=\{1\}$. So the solution exists for $t \in[0,1\rceil$.

So for all $\varphi(t)$ we can guarantee the existence of solution of the differential equation

$$
\begin{aligned}
& D X(t)=\Phi(\varphi(t))[1,3], \\
& X(0)=[2,4]
\end{aligned}
$$

on the interval $\lceil 0,1\rceil$.

Obviously, for example if $\varphi(t)=\sin t$ the solution exists on $[0,+\infty)$.

The following theorem of existence of the solution of equation (9) holds:

Theorem 1. Let the interval-valued mapping $F(t, X)$ in the domain $Q=\left\{(t, X): t \in\left[t_{0}, t_{0}+a\right], h\left(X, X_{0}\right) \leq b\right\}$ satis fy the conditions:

a) for any fixed $X$ the interval-valued mapping $F(\cdot, X)$ is measurable;

b) for almost every fixed $t$ the interval-valued mapping $F(t, \cdot)$ is continuous;

c) $|F(t, X)| \leq m(t)$, where $m(\cdot)$ is summable on $t \in\left[t_{0}, t_{0}+a\right]$;

d) $\varphi(t)$ has the finite number of intervals where $\operatorname{sign}(\varphi(t))= \pm 1$ on $\left[t_{0}, t_{0}+a\right]$.

Then there exists a solution of equation (9) defined on the interval $t \in\left[t_{0}, t_{0}+d\right]$, where $d>0$ satisfies the conditions

a) $d \leq a$;

b) $\phi\left(t_{0}+d\right) \leq b$, where $\phi(t)=\int_{t_{0}}^{t} m(s) d s$;

c) $\quad 2 \int_{\mu\left[t_{0}, t_{0}+d\right]} m(s) d s \leq \operatorname{diam} X_{0}=x_{20}-x_{10}$, where $\mu\left[t_{0}, t_{0}+d\right] \subset\left[t_{0}, t_{0}+d\right]: \varphi(t)<0$ for $t \in \mu\left[t_{0}, t_{0}+d\right]$.

Proof. Consider the behaviour of the function $\varphi(t)$ on the interval $\left[t_{0}, t_{0}+d\right]$. By condition $\mathrm{d}$ ) of the theorem there exists a finite number of points $t_{1}, \ldots, t_{m} \in\left(t_{0}, t_{0}+d\right)$, $t_{i}<t_{i+1}, t_{m+1}=t_{0}+d$ such that the function $\varphi(t)$ doesn't change its sign on the interval $\left(t_{i}, t_{i+1}\right), i=\overline{0, m}$. Suppose that there exists a solution of the equation (9) on the interval $\left[t_{0}, t_{k}\right], k=\overline{0, m}$. If $t_{k}<t_{0}+d$ consider the equation (9) on the interval $\left[t_{k}, t_{k+1}\right]$. 
Case $\quad \varphi(t)>0 . \quad$ Let $\quad X(t)=\left[x_{1}(t), x_{2}(t)\right]$, $F(t, X)=\left[f_{1}\left(t, x_{1}, x_{2}\right), f_{2}\left(t, x_{1}, x_{2}\right)\right]$. Then the interval- valued mapping $\left[x_{1}(t), x_{2}(t)\right]$ satisfies the integral equation

$$
\begin{aligned}
& {\left[x_{1}(t), x_{2}(t)\right]=\left[x_{1}\left(t_{k}\right), x_{2}\left(t_{k}\right)\right]+} \\
& +\int_{t_{k}}^{t}\left[f_{1}\left(s, x_{1}(s), x_{2}(s)\right), f_{2}\left(s, x_{1}(s), x_{2}(s)\right)\right] d s,
\end{aligned}
$$

i.e. the functions $x_{1}(t), x_{2}(t)$ satisfy the system of integral equations

$$
\left\{\begin{array}{l}
x_{1}(t)=x_{1}\left(t_{k}\right)+\int_{t_{k}}^{t} f_{1}\left(s, x_{1}(s), x_{2}(s)\right) d s, \\
x_{2}(t)=x_{2}\left(t_{k}\right)+\int_{t_{k}}^{t} f_{2}\left(s, x_{1}(s), x_{2}(s)\right) d s .
\end{array}\right.
$$

Therefore the functions $x_{1}(t), x_{2}(t)$ satisfy the system of differential equations

$$
\begin{cases}\dot{x}_{1}=f_{1}\left(t, x_{1}, x_{2}\right), & x_{1}\left(t_{k}\right)=x_{1}\left(t_{k}-0\right), \\ \dot{x}_{2}=f_{2}\left(t, x_{1}, x_{2}\right), & x_{2}\left(t_{k}\right)=x_{2}\left(t_{k}-0\right) .\end{cases}
$$

Using the Caratheodory theorem [9] we have that there exists a solution of this system defined on the interval $\left[t_{k}, t_{k+1}\right]$.

Case $\varphi(t)<0$. Then the interval-valued mapping $\left[x_{1}(t), x_{2}(t)\right]$ satisfies the integral equation

$$
\begin{aligned}
& {\left[x_{1}(t), x_{2}(t)\right]=} \\
& =\left[x_{1}\left(t_{k}\right), x_{2}\left(t_{k}\right)\right]-\int_{t_{k}}^{t}\left[f_{1}\left(s, x_{1}(s), x_{2}(s)\right), f_{2}\left(s, x_{1}(s), x_{2}(s)\right)\right] d s,
\end{aligned}
$$

i.e. the functions $x_{1}(t), x_{2}(t)$ satisfy the system of integral equations

$$
\left\{\begin{array}{l}
x_{1}(t)+\int_{t_{k}}^{t} f_{1}\left(s, x_{1}(s), x_{2}(s)\right) d s=x_{1}\left(t_{k}\right), \\
x_{2}(t)+\int_{t_{k}}^{t} f_{2}\left(s, x_{1}(s), x_{2}(s)\right) d s=x_{2}\left(t_{k}\right) .
\end{array}\right.
$$

Therefore the functions $x_{1}(t), x_{2}(t)$ satisfy the system of differential equations

$$
\begin{cases}\dot{x}_{1}=-f_{1}\left(t, x_{1}, x_{2}\right), & x_{1}\left(t_{k}\right)=x_{1}\left(t_{k}-0\right) \\ \dot{x}_{2}=-f_{2}\left(t, x_{1}, x_{2}\right), & x_{2}\left(t_{k}\right)=x_{2}\left(t_{k}-0\right) .\end{cases}
$$

Using the Caratheodory theorem we have that there exists a solution of this system defined on the interval $\left[t_{k}, t_{k+1}\right]$. Then there exists a solution of differential equation (9) provided that $\operatorname{diam} X(t) \geq 0$. The fact that $\operatorname{diam} X(t) \geq 0$ follows from the condition $\mathrm{c}$ ) of the theorem:

$$
\begin{aligned}
\operatorname{diam} X(t) & \geq \operatorname{diam} X\left(t_{k}\right)-2 \int_{\left[t_{k}, t\right]} m(s) d s \geq \\
& \geq \operatorname{diam} X_{0}-2 \int_{\mu\left[t_{0}, t\right]} m(s) d s \geq 0
\end{aligned}
$$

because on intervals where $\varphi(t) \geq 0 \quad \operatorname{diam} X(t)$ doesn't decrease.

3) $\varphi(t) \equiv 0$. Then we have $X(t) \equiv X\left(t_{k}\right)$. So the solution of differential equation (9) exists on $\left[t_{k}, t_{k+1}\right]$.

\section{Conclusions}

In this paper the concept of generalized differentiability (proposed in [17]) for interval-valued mappings is used. The interval-valued differential equations with generalized derivative are considered and the existence theorem is proved.

\section{REFERENCES}

[1] Ambrosio,L., and Tilli, L., 2004, Topics on analysis in metric spaces, Oxford Lecture Series in Mathematics and its Applications 25, Oxford University Press, Oxford.

[2] Bede, B., and Gal, S.G., 2004, Almost periodic fuzzy-number-valued functions, Fuzzy Sets Syst., 147(3), 385-403, doi:10.1016/j.fss.2003.08.004.

[3] Bede, B., and Gal, S.G., 2005, Generalizations of the differentiability of fuzzy-number-valued functions with applications to fuzzy differential equations, Fuzzy Sets Syst., 151, 581-599, doi:10.1016/j.fss.2004.08.001.

[4] Bede, B., and Stefanini L., 2008, Generalized Hukuhara differentiability of interval-valued functions and interval differential equations, Working Paper Series in Economics, Math. and Statistics, WP-EMS \#2008/03, Univ. Urbino "Carlo Bo".

[5] de Blasi, F.S., and Iervolino F., 1969, Equazioni differentiali con soluzioni a valore compatto convesso, Boll. Unione Mat. Ital., 2 (4 - 5), 491-501.

[6] de Blasi, F.S., and Iervolino F., 1971, Euler method for differential equations with set - valued solutions, Boll. Unione Mat. Ital., 4 (4), 941-949.

[7] de Blasi, F.S., Lakshmik antham, V., Gnana Bhaskar T., 2007, An existence theorem for set differential inclusions in a semilinear metric space, Control Cybernet., 36 (3), 571-582.

[8] Brandao Lopes Pinto, A.J., de Blasi, F.S., Iervolino, F., 1970, Uniqueness and existence theorems for differential equations with compact convex valued solutions, Boll. Unione Mat. Ital., 4, 534-538.

[9] Filippov, A.F., Differential equations with discontinuous right-hand side, 1985, Nauka, Moscow.

[10] Hukuhara, M.,1967, Integration des applications mesurables dont la valeur est un compact convexe, Funkcial. Ekvac., 10, 205-223.

[11] Lakshmikantham, V., Granna Bhaskar, T., Vasundhara Devi, J., 2006, Theory of set differential equations in metric spaces, Cambridge Scientific Publishers.

[12] Lakshmikantham, V., Mohapatra, R.N., 2003, Theory of Fuzzy Differential Equations and Inclusions, Taylor $1 \&$ Francis, London, doi:10.1201/9780203011386.

[13] Perestyuk, N. A., Plotnikov, V.A., Samoilenko, A.M., 
Skripnik, N.V., 2011. Differential equations with impulse effects: multivalued right-hand sides with discontinuities, De Gruyter Studies in Mathematics: 40, Berlin/Boston: Walter De Gruyter GmbH\&Co.

[14] Phu, N.D., Tung, T.T., 2005, Multivalued Differential Equations, VNU -HCM City: Publishing House.

[15] Plotnikov, A.V., 2000, Differentiation of multivalued mappings. T-derivative, Ukr. Math. J., 52 (8), 1282-1291, doi:10.1023/A:1010361206391.
[16] Plotnikov, A.V., and Skripnik, N.V., 2009, Differential equations with "clear" and fuzzy multivalued right-hand sides. Asymptotics Methods, AstroPrint, Odessa. (in Russian)

[17] Plotnikov, A.V., and Skripnik, N.V., 2011, Set-valued differential equations with generalized derivative, J. Adv. Res. Pure Math., 3 (1), 144 - 160, doi:10.5373/jarpm.475.062210.

[18] Plotnikov, V.A., Plotnikov, A.V., Vityuk, A.N., 1999, Differential equations with a multivalued right-hand side. Asymptotic methods, AstroPrint, Odessa. 